\title{
The Kidney, a Victim and Culprit of Autoimmune and Alloimmune Responses
}

\author{
Claudio Ponticelli $^{a}$ Maurizio Salvadori ${ }^{b}$ Rosanna Coppo ${ }^{c}$ \\ ${ }^{a}$ Nephrology and Dialysis, Istituto Scientifico Humanitas, Rozzano, ${ }^{\mathrm{b}}$ Nephrology, Dialysis and Transplantation, \\ Careggi University Hospital, Florence, and ' Nephrology, Dialysis and Transplantation, Regina Margherita University \\ Hospital, Turin, Italy
}

\section{Key Words}

Glomerulonephritis · Kidney transplantation · Therapy •

Pathogenesis $\cdot$ Immunonephrology Working Group

\begin{abstract}
The Working Group of the European Renal Association-European Nephrology and Dialysis Association (ERA-EDTA) dedicated to immune system involvement in renal disease (Immunonephrology Working Group) was established in 2009 to facilitate exchanges of ideas on basic science research and new treatment protocols among European nephrologists. A section of the ERA-EDTA website describes activities and is open for new applications and proposals. In 2010, the first meeting of this Working Group focused on aspects of immune-mediated renal damage shared by kidney during glomerulonephritides or after kidney transplantation. From the large series of data reported, a previously disregarded presence of pathogenetic and therapeutic aspects common to glomerular diseases and transplanted kidneys was highlighted. Although the antigens involved in transplant rejection and in glomerulonephritis are different, a number of factors involved in the effector response are similar, mainly those based on the interaction between innate and adaptive immune mechanisms and on the strict cooperation between T and $\mathrm{B}$ cells. Moreover, the common target for the allo- and autoimmune attacks is represented by the endothelial cells,
\end{abstract}

of which the kidney is particularly rich. These similarities may explain why immunomodulating treatments used in alloimmunity may also be useful in autoimmune diseases and vice versa. Considering renal damage from a holistic point of view may favour exchange of news for two formerly considered non-communicant areas, i.e. glomerular disease and renal transplantation nephrology sections.

Copyright $\odot 2011$ S. Karger AG, Basel

An international meeting focused on immune-mediated renal damage was organized last May in Florence under the patronage of the European Renal AssociationEuropean Nephrology and Dialysis Association (ERAEDTA) Immunonephrology Working Group. During this two-day meeting some common pathogenetic and therapeutic aspects shared by glomerulonephritis (GN) and kidney transplantation were lively discussed. The meeting focused on the roles of innate immunity and B cells in auto- and alloimmune responses and on some recent therapeutic approaches in GN and renal transplantation.

There is now solid evidence that the innate immunity not only protects the organism from infection but can also activate the adaptive immunity through antigen presenting cells (APC) that present self- or alloantigens to T and B cells. The role of three main players was analyzed in depth, namely complement, Toll-like receptors (TLRs),

\section{KARGER}

Fax +41613061234 E-Mail karger@karger.ch www.karger.com (c) 2011 S. Karger AG, Basel

$1660-2110 / 11 / 1193-0200 \$ 38.00 / 0$

Accessible online at:

www.karger.com/nec
Rosanna Coppo, MD

Nephrology, Dialysis and Transplantation

Regina Margherita University Hospital, Piazza Polonia 94

IT-10126 Torino (Italy)

E-Mail rosanna.cappo@unito.it 
and dendritic cells (DCs) $[1,2]$. Dr. Daha focused on complement activation in GN. He pointed out that complement can be activated via the classical pathway in some immune complex-mediated GN [3], such as cryoglobulinaemic and lupus nephritis, while in dense deposit disease and atypical haemolytic uraemic syndrome (and possibly also in other GNs and some vasculitides) there is uncontrolled activation of the alternative pathway due to genetic downregulation of complement controllers such as factor H. In IgA nephropathy, the lectin pathway of complement results were activated. On the other hand, not only an overactivity of complement cascade, but also a deficient production of some complement components, i.e. C1q, can trigger the development of lupus nephritis due to a reduced uptake of apoptotic cells by immature DCs to clear cellular debris and to inhibited production of interferon- $\alpha$ by DCs $[4,5]$.

Dr. Regele reviewed the role of complement activation in antibody-mediated rejection (AMR). Capillary C4d deposition is now considered as a reliable marker of AMR, having a high level of sensitivity and specificity [6]. Only in a few cases can C4d deposits be detected even in the absence of circulating antibodies [7]. A possible strategy for an early recognition of a C4d-positive AMR may consist in monitoring the presence of donor-specific alloantibodies (DSA) in the serum performing a renal biopsy whenever circulating DSA are detected.

In infections, microbial components provide signals that alert the immune system to danger and promote the generation of immunity [8]. Danger signals are thought to act by stimulating DCs to mature so that they can present foreign antigens and stimulate $\mathrm{T}$ lymphocytes. Dr. Anders discussed the potential pathogenetic role of TLRs, a family of innate immune-recognition receptors expressed on DCs which detect danger signals. He pointed out that activation of the innate immune system can be triggered not only by inflammation caused by pathogens, but also by tissue damage caused by metabolic, haemodynamic, toxic, or autoimmune injuries. TLR activation is unable to eliminate the underlying drivers of autoimmunity, rather it can induce cytokine and chemokine secretion, leukocyte recruitment, and tissue remodelling, becoming a maladaptive pathogenic mechanism that aggravates renal damage. In turn, autophagy allows stressed cells to reduce intracellular microorganisms, protein aggregates, and cellular organelles by digesting them in autophagolysosomes [9]. Within the autophagolysosome, endogenous molecules and danger-associated molecules may be presented to TLRs or loaded onto the major histocompatibility complex and presented as autoantigens.
Thus, danger signalling may represent a common mechanism responsible for kidney injury in $\mathrm{GN}$, as reported in experimental lupus nephritis [10]. Also in humans with IgA nephropathy, increased TLR expression on peripheral mononuclear cells suggests activation of a danger signal of innate immunity [11].

Dr. Castellano showed that renal infiltrating DCs may favour the local synthesis of $\mathrm{Clq}$ and complement activation in lupus nephritis [12], so facilitating the engulfment of apoptotic renal cells and the presentation of autoantigens to the adaptive immune response. In ischaemia-reperfusion injury, damage-associated molecules, such as heat-shock proteins, can act as endogenous ligands for donor- and recipient-derived TLR-4- and TLR 2-bearing DCs. After this recognition, DCs mature and initiate the recipient's adaptive alloimmune response leading to fully expressed ischaemia-reperfusion injury. Complement also plays an important role in this condition. Even a short ischaemia time can activate the classical and lectin pathways of complement as shown by peritubular capillary and glomerular C4d and C5b-9 deposition.

A typical model of GN caused by circulating antibodies is membranous nephropathy (MN). The group of Dr. Ronco identified specific antibodies against neutral endopeptidase in newborns from mothers deficient in these podocyte enzymes [13]. Antibodies directed against $\mathrm{M}$ type phospholipase-2 receptors (PLA2R) and aldose reductase and manganese superoxide dismutase have also been detected in adults with idiopathic MN [14]. The expression of these autoantigens in podocytes can trigger the production of specific antibodies (mainly $\operatorname{IgG}_{4}$ ) and deposits of immune complexes on the epithelial side of the glomerular basement membrane. Some exogenous antigens might also participate in the development of idiopathic MN. Dr. Ronco hypothesized that exogenous cationic proteins, such as bovine serum albumin, may be reabsorbed by the gastrointestinal mucosa, enter the circulation, and be attracted by the anionic charge on the epithelial side of the glomerular basement membrane where they may be reached by antibodies. The mechanisms leading to recurrent $\mathrm{MN}$ after renal transplantation are still far from being elucidated. Considering the recent evidence that podocyte enzymes represent the antigens in the original disease, one might speculate that recurrent $\mathrm{MN}$ is triggered by autoantibodies from the circulation with conformational epitopes exposed or genetically determined in the podocytes of the donor kidney [15].

For many years, in renal transplantation the attention has been mainly focused on cellular immunity, however 
a number of kidney allografts fail as a consequence of an AMR. The still open question in renal transplantation (but also in lupus nephritis and vasculitis) is whether the antibodies we detect in the serum of the recipient are actually those responsible of tissue damage. Dr. Cozzi pointed out that antibodies directed against the antigens expressed by the endothelium of the graft can damage the graft by eliciting endothelial cell injury via complementdependent mechanisms and by stimulating inflammatory and proliferation signals [16]. To support this concept, Dr. Cozzi designed a prospective trial to evaluate the possible benefit of removing circulating antibodies by plasmapheresis and intravenous immunoglobulins. The results of this trial could clarify whether the anti-donor antibodies we detect in the circulation are the same as those causing graft injury.

An interesting new target for blunting pathogenetic mechanisms operating in some GNs and in particular settings of grafted kidney is NF- $\mathrm{B}$, an ubiquitous transcriptor factor which governs the expression of genes encoding for cytokines, growth factors, adhesion molecules and other factors involved in the immune and inflammatory response. Dr. Coppo showed that NF- $\kappa \mathrm{B}$ is activated in renal tissue and in peripheral blood mononuclear cells of patients with IgA nephropathy [17] or focal segmental glomerulosclerosis. She suggested that since NF-кB nuclear translocation is regulated by the ubiquitin-proteasome pathway, it would be possible to use proteasome inhibitors in cases of severe nephrotic syndrome or lupus nephritis resistant to standard treatments. In transplantation, proteasome inhibitors such as bortezomib have recently provided interesting results in antibody-mediated acute kidney rejection $[18,19]$.

Dr. Egido reported some promising results with the use of suppressors of cytokine signalling (SOCS). These are intracellular proteins that work as negative regulators for Janus kinase/signal transducer and activator of transcription (JAK/STAT) signalling pathways [20]. Activation of JAK/STAT is an important mechanism by which hyperglycaemia contributes to renal damage. In a rat model of diabetes with JAK/STAT activation, the intrarenal delivery of adenovirus expressing SOCS1 and SOCS3 significantly improved renal function and reduced renal lesions [21]. SOCS gene delivery also decreased the activation of STAT1 and STAT3, and the expression of proinflammatory and profibrotic proteins in the diabetic kidney. These results suggest that suppression of the JAK/ STAT pathway, by increasing intracellular SOCS proteins, may have therapeutic potential in diabetic nephropathy and perhaps also in other progressive renal diseases. Since the tyrosine kinase JAK-3 transduces cytokine-mediated signals to phosphatidylinositol kinase-3, which provides the signal for lymphocyte proliferation by activating a cascade of different kinases, SOCS may probably play an important role also in preventing rejection of renal transplantation.

Dr. Ostendorf introduced the concept that renal fibrosis should be considered as a dynamic system that involves extracellular matrix components and many renal and infiltrating cells [22]. He demonstrated that the involved cells exhibit enormous plasticity or phenotypic variability, and pointed out that further studies are needed to better understand the underlying mechanisms of renal fibrosis in native kidney diseases as well as in failing transplants and to find effective treatments. Dr. Ostendorf also discussed the central role of platelet-derived growth factor (PDGF) in experimental mesangioproliferative GN and pointed out that patients with IgA nephropathy, particularly those with an early disease, have very high serum levels of PDGF-DD [23]. Thus, serum PDGF-DD may be considered a good biomarker of this disease and a possible target for future therapies.

Dr. Campistol reviewed the results with the use of two inhibitors of mTOR, sirolimus and everolimus, in renal transplantation [24]. The use of these immunosuppressive drugs can allow reducing the dosage and the adverse events of calcineurin inhibitors (CNI). However, in patients with creatinine clearance $<40 \mathrm{ml} / \mathrm{min}$ or with proteinuria $>800 \mathrm{mg} / \mathrm{day}$, the switch from CNI to antimTOR can cause a deterioration of renal allograft function and proteinuria. The interest of mTOR inhibitors also rests on their pleiotropic effects. They can inhibit the replication of some herpes viruses (cytomegalovirus, Epstein-Barr virus, human herpes virus 8), can inhibit the arterial intimal proliferation, and can exert anti-oncogenic effects by inhibiting vascular endothelial growth factors, and some oncogenes.

Dr. Grinyo reminded that to be activated, T cells require a dual signal. The first signal is given by the contact between the peptide of the HLA system presented by APC and a receptor of $\mathrm{T}$ cell. The second signal (co-stimulation) is provided by the interaction between molecules expressed on the membrane of APC and on the T cell. The best characterized T-cell co-stimulatory pathway involves the CD28 receptor, which binds to two co-stimulatory molecules, B7-1 and B7-2. CTLA-4, an antigen similar to CD28, blocks the co-stimulation pathway and inhibits interleukin-2 production and cell cycle progression. Different approaches have been attempted to interfere with the co-stimulation pathway, such as anti-CD80/86 
Table 1. Synoptic table summarizing pathogenetic mechanisms operating in both glomerular diseases (GN) and renal transplant damage

\begin{tabular}{|c|c|c|}
\hline Pathogenetic mechanism & Glomerulonephritis & Kidney transplant damage \\
\hline Complement activation & $\begin{array}{l}\text { Lupus nephritis, post-infectious GN, cryoglobu- } \\
\text { linaemic GN, atypical haemolytic uraemic } \\
\text { syndrome }\end{array}$ & AMR (CD4 perivascular deposits) \\
\hline $\begin{array}{l}\text { TLR ligation and } \\
\text { DC activation }\end{array}$ & Lupus nephritis, IgA nephropathy & $\begin{array}{l}\text { Alloimmune response in grafted } \\
\text { kidneys }\end{array}$ \\
\hline Circulating antibodies & Autoimmune GN, membranous GN & AMR \\
\hline Transcription factors (NF- $\kappa \mathrm{B})$ & Nephrotic syndrome, IgA GN, lupus nephritis & AMR \\
\hline $\begin{array}{l}\text { Transcription factor JAK/STAT } \\
\text { cytokine signalling }\end{array}$ & Experimental diabetes & Rejection injury \\
\hline Renal fibrosis & GN progression & Failing transplant \\
\hline Co-stimulatory molecules & Lupus nephritis & Renal graft rejection \\
\hline B-cell activation & Lupus nephritis, vasculitis & AMR \\
\hline $\begin{array}{l}\text { Targeted therapy to one or more } \\
\text { of the above mechanisms }\end{array}$ & $\begin{array}{l}\text { Under study for application or already in use in } \\
\text { glomerular diseases }\end{array}$ & $\begin{array}{l}\text { Under study for application or } \\
\text { already used in renal transplantation }\end{array}$ \\
\hline
\end{tabular}

monoclonal antibody (MAB) and CTLA4-linked immunoglobulins (abatacept and belatacept) [25]. The clinical experience in kidney transplantation showed that belatacept prevented T-cell activation and reduced humoral alloresponses, it was associated with low rates of rejection, did not cause nephrotoxicity, and ameliorated the cardiovascular profile when compared to CNI. However, cases of lymphoma were reported in EBV-negative patients. Experimental and preliminary clinical studies suggested a possible role of these agents also in lupus nephritis. Randomized, controlled trials are under way.

Dr. Jayne discussed the array of MABs directed against membrane proteins expressed on B cells. Among them, the more frequently used in autoimmune diseases is rituximab, a chimeric $\mathrm{MAB}$ that selectively targets $\mathrm{CD} 20+\mathrm{B}$ cells. Good results have been reported in patients with idiopathic nephrotic syndrome, HCV-related cryoglobulinaemia, lupus nephritis, and vasculitis. However, large randomized controlled trials could not demonstrate the superiority of rituximab over standard treatment either in patients with lupus nephritis or in those with renal vasculitis, with the exception of granulomatosis with polyangitis (Wegener's) [26].

Dr. Salvadori discussed the relevance of the antibody network in renal transplantation. DSA, either preformed or developed after transplantation, can be responsible for severe forms of acute and chronic rejection. Antibodies can be removed by intense plasmapheresis. It is also possible to interfere with their pathogenetic mechanisms by high-dose intravenous immunoglobulins, mainly if enriched with sialic acid, or by the MAB eculizumab that interferes with $\mathrm{C} 5$ activation. Finally, elimination of antibody-producing cells can be obtained by bortezomib, a proteasome inhibitor [27], rituximab and some new generation drugs, including fully human anti-CD20 drugs and drugs targeting BAFF or BAFF receptor.

In conclusion, there is increasing evidence showing that the alloimmune responses to HLA antigens and the autoimmune responses to self-antigens appear to share a number of common pathways (table 1). Innate immunity plays a major role in activating adaptive immunity both in renal transplant rejection and in different types of primary or secondary GN, as demonstrated by the deep involvement of TLRs, DCs, and complement. Moreover, it should be pointed out that if renal transplant recipients and patients with GN use different mechanisms (although with some similarities) to increase the immune response to different antigens, the chain of events eventually leading to inflammation and tissue damage is the same in alloimmunity and in autoimmunity. This can explain why drugs initially designed to interfere with the autoimmune response can be successfully used in renal transplantation and vice versa. 


\section{References}

$\checkmark 1$ Allam R, Anders HJ: The role of innate immunity in autoimmune tissue injury. Curr Opin Rheumatol 2008;20:538-544.

$\checkmark 2$ Obhrai J, Goldstein DR: The role of Toll-like receptors in solid organ transplantation. Transplantation 2006;81:497-502.

$\checkmark 3$ Chen M, Daha MR, Kallenberg CG: The complement system in systemic autoimmune disease. J Autoimmun 2010;34:J276J286.

4 Li M, O’Sullivan KM, Jones LK, et al: CD100 enhances dendritic cell and CD4+ cell activation leading to pathogenetic humoral responses and immune complex glomerulonephritis. J Immunol 2006;177:3406-3412.

5 Lood C, Gullstrand B, Truedsson L, et al: C1q inhibits immune complex-induced interferon- $\alpha$ production in plasmacytoid dendritic cells: a novel link between C1q deficiency and systemic lupus erythematosus pathogenesis. Arthritis Rheum 2009;60:3081-3090.

66 Batal I, Girnita A, Zeevi A, et al: Clinical significance of the distribution of C4d deposits in different anatomic compartments of the allograft kidney. Mod Pathol 2008;21:14901498.

7 Haas M: The significance of C4d staining with minimal histologic abnormalities. Curr Opin Organ Transplant 2010;15:21-27.

$\checkmark 8$ Anders HJ, Zecher D, Schlöndorff D: Microbial nucleic acids in the pathogenesis of glomerulonephritis. Nephrol Ther 2006;2:422431.

-9 Anders H-J, Schlondorff DO: Innate immunity receptors and autophagy: implications for autoimmune kidney injury. Kidney Int 2010;78:29-37.

10 Lartigue A, Colliou N, Calbo S, et al: Critical role of TLR2 and TLR4 in autoantibody production and glomerulonephritis in lpr mutation-induced mouse lupus. J Immunol 2009; 183:6207-6212.
1 Coppo R, Camilla R, Amore A, et al: Toll-like receptor-4 expression is increased in circulating mononuclear cells of patients with immunoglobulin A nephropathy. Clin Exp Immunol 2010;159:73-81.

$\checkmark 12$ Fiore N, Castellano G, Blasi A, et al: Immature myeloid and plasmacytoid dendritic cells infiltrate renal tubulointerstitium in patients with lupus nephritis. Mol Immunol 2008;45:259-265.

13 Debiec H, Guigonis V, Mougenot B, et al: Antenatal membranous glomerulonephritis due to anti-neutral endopeptidase antibodies. N Engl J Med 2002;346:2053-2060.

14 Beck LH Jr, Bonegio RG, Lambeau G, et al: M-type phospholipase $\mathrm{A}_{2}$ receptor as target antigen in idiopathic membranous nephropathy. N Engl J Med 2009;361:11-21.

15 Ponticelli C, Glassock RJ: Posttransplant recurrence of primary glomerulonephritis. Clin J Am Soc Nephrol 2010;5:23632372.

16 Seveso M, Bosio E, Ancona E, Cozzi E: De novo anti-HLA antibody responses after renal transplantation: detection and clinical impact. Contrib Nephrol. Basel, Karger, 2009, vol 162, pp 87-98.

17 Coppo R, Camilla R, Alfarano A, et al: Upregulation of the immunoproteasome in peripheral blood mononuclear cells of patients with IgA nephropathy. Kidney Int 2009;75: 536-541.

18 Flechner SM, Fatica R, Askar M, et al: The role of proteasome inhibition with bortezomib in the treatment of antibody-mediated rejection after kidney-only or kidney-combined organ transplantation. Transplantation 2010;90:1486-1492.

19 Walsh RC, Everly JJ, Brailey P, et al: Proteasome inhibitor-based primary therapy for antibody-mediated renal allograft rejection. Transplantation 2010;89:277-284.
20 Gómez-Guerrero C, López-Franco O, et al: Suppressors of cytokine signaling regulate Fc receptor signaling and cell activation during immune renal injury. J Immunol 2004; 172:6969-6977.

21 Ortiz-Muñoz G, Lopez-Parra V, LopezFranco O, et al: Suppressors of cytokine signaling abrogate diabetic nephropathy. J Am Soc Nephrol 2010;21:763-772.

22 Eitner F, Bücher E, van Roeyen C, et al: PDGF-C is a proinflammatory cytokine that mediates renal interstitial fibrosis. J Am Soc Nephrol 2008;19:281-289.

23 Boor P, Eitner F, Cohen CD, et al: Patients with IgA nephropathy exhibit high systemic PDGF-DD levels. Nephrol Dial Transplant 2009;24:2755-2762.

24 Russ G, Segoloni G, Oberbauer R, et al: Superior outcomes in renal transplantation after early cyclosporine withdrawal and sirolimus maintenance therapy, regardless of baseline renal function. Transplantation 2005;80: 1204-1211.

25 Vincenti F, Charpentier B, Vanrenterghem $\mathrm{Y}$, et al: A phase III study of belatacept-based immunosuppression regimens versus cyclosporine in renal transplant recipients (BENEFIT study). Am J Transplant 2010;10:535546.

26 Jones RB, Ferraro AJ, Chaudhry AN, Brogan P, Salama AD, Smith KG, Savage CO, Jayne DR: A multicenter survey of rituximab therapy for refractory antineutrophil cytoplasmic antibody-associated vasculitis. Arthritis Rheum 2009;60:2156-2168.

27 Kaposztas Z, Podder H, Mauiyyedi S, et al: Impact of rituximab therapy for treatment of acute humoral rejection. Clin Transplant 2009;23:63-73. 